The aim of this clinical study was to investigate the effect of nebulised GSK1995057 on pulmonary and systemic inflammation and cell injury in an in vivo human model of lung injury induced by inhaled lipopolysaccharide (LPS).

Methods Healthy subjects were enrolled in a double-blind, placebo-controlled study and randomised to nebulised GSK1995057 or placebo (1:1) administered 1 hour prior to LPS inhalation. Measurements were performed in bronchoalveolar lavage (BAL) fluid obtained at 6 hours after LPS challenge (7 hours after dosing) and in serum obtained over 24 hours post dosing of GSK1995057. The primary endpoint was BAL neutrophil count at 6 hours post LPS exposure. Data are geometric mean (95\% CI).

Results Thirty-seven healthy subjects were enrolled. One subject in the placebo group was excluded from the analysis of BAL markers as the BAL was technically poor. Pre-treatment with inhaled GSK1995057 significantly reduced pulmonary and systemic markers of inflammation. In addition, there was a reduction in pulmonary vWF reflecting reduced endothelial cell injury/activation (Table 1). The prevalence of LPS-induced clinical symptoms (e.g. fever, nausea) was also lower in GSK1995057 treated subjects compared with placebo treated subjects. There were no serious adverse events related to study drug.

Conclusion This is the first report that inhalation of a novel human antibody fragment directed against the TNFR1 receptor attenuates mechanisms implicated in the pathophysiology of ALI. GSK1995057 may be a potential therapy for ALI.

ClinicalTrials.gov identifier: NCT01587807.

This work was funded by GlaxoSmithKline.

\begin{tabular}{|c|c|c|c|c|}
\hline BALF & $\begin{array}{l}\text { Placebo } \\
(\mathrm{n}=18)\end{array}$ & $\begin{array}{l}\text { GSK1995057 } \\
(n=18)\end{array}$ & $\begin{array}{l}\% \\
\text { reduction }\end{array}$ & P-value \\
\hline PMN & $6.5(4.5,9.4)$ & $4.5(2.89,6.89)$ & $31(41)^{\dagger}$ & $0.17(0.03)^{\dagger}$ \\
\hline$\left(10^{4}\right.$ cells $\left./ \mathrm{ml}\right)$ & & $3.8(2.8,5.3)^{*}$ & & \\
\hline $\mathrm{IL}-1 \mathrm{~b}(\mathrm{pg} / \mathrm{ml})$ & $6.8(4.3,10.4)$ & $1.4(1.0,2.1)$ & 79 & $<0.0001$ \\
\hline IL-6 (pg/ml) & $386.1(277.5,537.4)$ & $169.4(111.2,257.9)$ & 56 & 0.003 \\
\hline IL-8 (pg/ml) & $332.1(254.6,433.2)$ & $117.5(82.5,167.54)$ & 65 & $<0.0001$ \\
\hline MIP1alpha & $133.7(87.2,205.1)$ & $15.6(8.3,29.1)$ & 88 & $<0.0001$ \\
\hline \multicolumn{5}{|l|}{$(\mathrm{pg} / \mathrm{ml})$} \\
\hline MCP-1 (pg/ml) & $799.4(591.6,1080.2)$ & $159.5(101.9,249.5)$ & 80 & $<0.0001$ \\
\hline vWF (ng/ml) & $12.8(9.2,17.9)$ & $8.1(6.1,10.8)$ & 37 & 0.04 \\
\hline Serum & $\begin{array}{l}\text { Placebo } \\
(\mathrm{n}=19)\end{array}$ & $\begin{array}{l}\text { GSK1995057 } \\
(n=18)\end{array}$ & $\begin{array}{l}\% \\
\text { reduction }\end{array}$ & P-value \\
\hline$\overline{C R P}(\mu \mathrm{g} / \mathrm{ml})^{* *}$ & $55.2(31.0,98.4)$ & $12.0(6.6,21.8)$ & 78 & 0.0007 \\
\hline OSM $(\mathrm{pg} / \mathrm{ml})^{* * * *}$ & $20.7(13.4,32.0)$ & $7.5(4.8,11.7)$ & 64 & 0.002 \\
\hline
\end{tabular}

*PMN data with subject classified as biological outlier $(>3 \mathrm{x}$ inter quartile range outside the upper quartile) removed.

to Reduction and statistical significance for BALPMN data with biological outlier excluded.

** CRP data taken at 24h post GSK1995057 dosing. Data are adjusted means with baseline and time effects considered.

${ }^{* * *}$ OSM data taken at $6 \mathrm{~h}$ post LPS inhalation. Data are adjusted means with baseline and time effects considered.

\section{EXPLOITING THE IMMUNOREGULATORY ROLE OF SIGLEC-E VIA SIALIC ACID-FUNCTIONALISED NANOPARTICLES AS A NOVEL APPROACH FOR THE TREATMENT OF ACUTE LUNG INJURY}

MK Greene, S Spence, F Fay, D Small, D Schmid, J Jaworski, JF Burrows, CM O'Kane, A Kissenpfennig, DF McAuley, CJ Scott; Queen's University Belfast, Belfast, United Kingdom

10.1136/thoraxjnl-2013-204457.102
Acute Lung Injury (ALI) is a life-threatening disorder underpinned by dysregulated inflammatory cascades, with resultant injury to lung architecture. Currently, provision of supportive care represents the mainstay of treatment for ALI and novel anti-inflammatory therapeutic strategies are urgently required. We have developed a polymeric nanoconstruct surface-functionalised with sialic acid targeting moieties (SNP), exploiting the anti-inflammatory effects arising from the targeted engagement of Siglec-E receptors on activated macrophages, with potential therapeutic utility in ALI.

Polylactic-co-glycolic acid (PLGA) nanoparticles of uniform size distribution (approximately 150nm in diameter) were synthesised in accordance with a salting-out formulation. Intratracheal instillation of $20 \mu \mathrm{g}$ lipopolysaccharide (LPS) was utilised as a model of ALI in C57BL/6 mice, co-administered with $1 \mu \mathrm{g}$ SNP or non-functionalised nanoparticles (NP). Bronchoalveolar lavage (BALF) samples were collected 24 hours after treatment for analysis by enzyme-linked immunosorbent assay (ELISA).

As exemplified in Figure 1., intratracheal instillation of SNP significantly attenuated BALF levels of pro-inflammatory TNF $\alpha$ and IL- 6 cytokines, in addition to the neutrophil chemoattractant KC. Moreover, BALF differential cell counts revealed a decrease in neutrophil numbers upon treatment with SNP under LPS-induced pro-inflammatory conditions. Further analyses addressing the therapeutic utility of SNP have been undertaken, including lung wet/dry ratios, histology and toxicological evaluation, with promising outcomes.

This research clearly demonstrates the ability of SNP to diminish the inflammatory response in a murine model of LPSinduced ALI. Considering that chemoattractants and cytokines are key mediators in the pathogenesis of ALI, these results substantiate the credibility of this nanoscaffold as a therapy for ALI. Ultimately, we aim to progress this modality to a human setting, specifically analysing its effects on alveolar macrophages isolated from human volunteers, before advancing to a human ex vivo lung perfusion model.
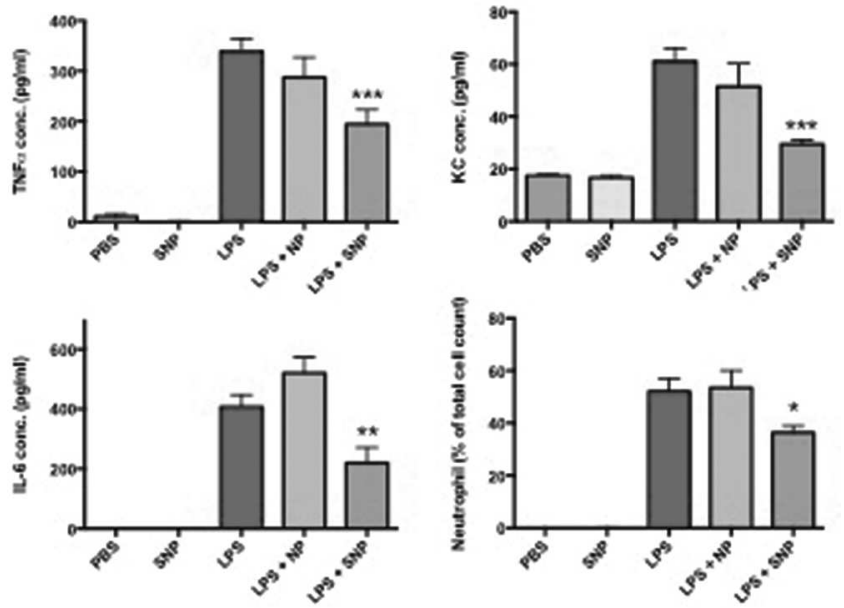

Abstract S95 Figure 1. Therapeutic efficacy of SNP in a murine model of LPS-induced ALI $(* p<0.05, * * p<0.001, * * * p<0.001$ compared to LPS control, as established by one-way ANOVA and Tuley post-hoc test).

\section{S96 SIMVASTATIN AS AN ADJUVANT THERAPY FOR INFECTION AND SEPSIS-IN-VITRO AND IN-VIVO STUDIES SUGGEST PRE-EMPTIVE / EARLY THERAPY IN THE ELDERLY}

JM Patel, H Greenwood, G Walton, F Gao, JM Lord, E Sapey, DR Thickett; Centre for Translational and Inflammatory Research, School of Clinical and Experimental 
Medicine, College of Medical and Dental Sciences, University of Birmingham, Birmingham, UK

\subsection{6/thoraxjnl-2013-204457.103}

Ageing is associated with increased episodes of sepsis and poorer outcomes. Statins are associated with improved outcomes during infection. We aimed to characterise the impact of age and acute severe infection on key neutrophil functions, assess whether physiologically relevant doses of simvastatin altered neutrophil functions and if benefits were seen, when during a septic episode statins could be utilised.

Methods Neutrophils from healthy volunteers and patients with lower respiratory tract infections (LRTI), pneumonia and sepsis were assessed for migratory accuracy, phagocytosis and neutrophil extracellular trap production before and after in-vitro treatment with simvastatin. Healthy elderly volunteers received $80 \mathrm{mg}$ simvastatin or placebo in a cross over double-blind randomised controlled trial and neutrophil functions were assessed. Data presented is for migration.

Results Neutrophils from healthy subjects ( $\mathrm{n}=70$, aged 21-94) demonstrated preserved neutrophil movement) $\left(\mathrm{R}^{2}=-0.48, \mathrm{p}<\right.$ 0.0001 ) towards chemoattractants (data shown for IL-8). Neutrophil chemotaxis decreased after 60yrs (comparing $<35$ to $>65 \mathrm{yrs}$ : mean difference (MD) $1.25 \mu \mathrm{m} / \mathrm{min}, \mathrm{p}=0.02$ ).

There was a progressive decrease in neutrophil chemotaxis in old patients with a LRTI, pneumonia and severe sepsis (MD compared to healthy control; LRTI $(\mathrm{n}=10), 0.7 \mu \mathrm{m} / \mathrm{min}$, $\mathrm{p}=0.04$; pneumonia $(\mathrm{n}=5), \mathrm{MD} 1.1 \mu \mathrm{m} / \mathrm{min}, \mathrm{p}=0.02$; sepsis $(\mathrm{n}=22) \mathrm{MD} 1.6 \mu \mathrm{m} / \mathrm{min}, \mathrm{p}=0.01)$ with "septic neutrophils" unable to mount targeted chemotaxis. Improvements to baseline were seen following recovery.

In-vitro treatment of neutrophils from healthy older people with simvastatin $(1 \mu \mathrm{M})$ restored "old" neutrophil chemotaxis to that of "young" cells. Simvastatin also restored neutrophil migration from old patients with LRTI and pneumonia to baseline but not in patients with sepsis.

Two weeks of oral simvastatin $80 \mathrm{mg}$ once daily therapy in healthy old volunteers (Age $>65, \mathrm{n}=20$ ) increased the accuracy of neutrophil migration $(\mathrm{MD} 1.68 \mu \mathrm{m} / \mathrm{min}, \mathrm{p}=0.02)$ replicating benchwork.

Conclusions "Elderly" neutrophil function is compromised in health, and deteriorates during infective episodes, in accordance with the severity of the insult. Migratory accuracy can be improved with simvastatin therapy however neutrophil function

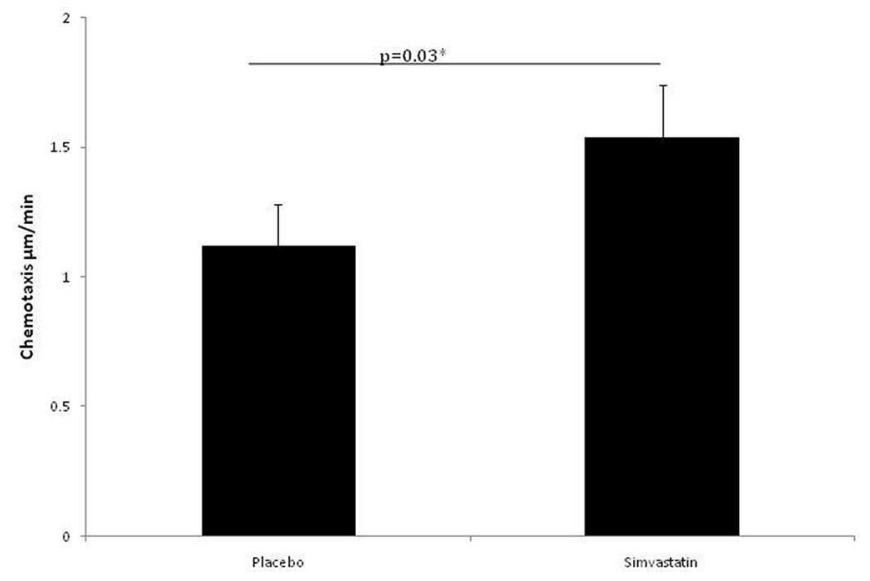

Abstract S96 Figure 1. Simavastatin 80mg once daily for 14 days improves directional migration (chemostaxis) of neutrophils from healthy elderly volunteers towards IL-8. *Student's t-Test in sepsis patients cannot be modulated during short term in-vitro therapy. Our data suggest statin therapy might be a preventative or an early adjuvant intervention rather than a treatment in established sepsis. We are testing whether simvastatin $80 \mathrm{mg}$ for seven days modifies neutrophil responses in elderly patients with pneumonia and sepsis (SNOOPI Trial).

\section{S97 ALVEOLAR EPITHELIAL DNA DAMAGE, INFLAMMATION AND ALTERED AUTOPHAGY FOLLOWING EXPOSURE TO SILVER NANOPARTICLES IS EXACERBATED BY VIRAL LIGANDS IN VITRO}

MZ Zambianchi, TD Tetley, AJ Thorley; Imperial College, London, England

\subsection{6/thoraxjn-2013-204457.104}

Rationale Over the past decade there has been a rapid increase in the development and use of engineered nanoparticles. Silver (Ag) is the most commercialised nanomaterial in the world. Due to its antibacterial activity it is incorporated into a variety of consumer goods including silver nanoparticle sprays to treat pulmonary infection. However, little is known about silver nanoparticle (AgNPs) toxicity in the lung. We hypothesise that inhalation of AgNPs will trigger a pro-inflammatory response in the alveolar epithelium, which, in the presence of viral infection, will synergise with the innate immune response, leading to increased pulmonary epithelial inflammation, DNA damage and autophagy activation.

Methods Transformed human alveolar epithelial type-1-like cells (TT1) were exposed to AgNPs alone and in combination with Poly I:C, a synthetic analogue of double strand RNA that mimics viral infection. Oxidative stress level was measured by dihydroethidium staining. Levels of IL-6 and IL- 8 were assessed by ELISA. DNA damage and autophagy marker LC3II/LC3I ratio were measured by western blot.

Results AgNPs induced oxidative stress in TT1 cells leading to enhanced inflammation, DNA double strand breakage and autophagy activation. The combination of $\mathrm{Ag}$ and Poly I:C potentiated IL-6 release (4-fold; $p<0.01$ ) and DNA damage (3fold; $\mathrm{p}<0.01$ ). Autophagy flux, activated by AgNPs alone, was slowed down by combined AgNPs and Poly I:C exposure.

Conclusion This study shows that Ag could induce oxidative stress in the lung, leading to a strong pro-inflammatory response and DNA damage, both potentiated by co-exposure to Poly I:C. Also, defective autophagy might result in certain human diseases such as cancer and neurodegenerative disease. This suggests that inhalation of AgNPs may have adverse health effects on the lung, that might be exacerbated by viral infection.

\section{S98 VITAMIN D DEFICIENCY INCREASES BACTERIAL LOAD IN} A MURINE MODEL OF SEPSIS-INDUCED LUNG INJURY

${ }^{1} \mathrm{D}$ Parekh, ${ }^{2} \mathrm{RCA}$ Dancer, ${ }^{2} \mathrm{~S}$ Lax, ${ }^{1} \mathrm{GD}$ Perkins, ${ }^{2} \mathrm{DR}$ Thickett; ${ }^{1}$ University of Warwick, Warwick, UK; ${ }^{2}$ University of Birmingham, Birmingham, UK

\subsection{6/thoraxjnl-2013-204457.105}

Introduction We have previously shown that patients with Acute Lung Injury (ALI) are severely vitamin D deficient. Several studies have reported a high prevalence of vitamin D deficiency in critically ill patients with sepsis, associated with increased morbidity and mortality but whether this is cause or effect is unknown. Bacteraemic sepsis is more common in the winter 